\title{
Final states of decaying 2D turbulence in bounded domains: Influence of the geometry
}

\author{
Kai Schneider ${ }^{a}, *$, Marie Farge ${ }^{\mathrm{b}}$ \\ ${ }^{a}$ M2P2-CNRS \& CMI, Université de Provence, Marseille, France \\ ${ }^{\mathrm{b}}$ LMD-CNRS, Ecole Normale Supérieure, Paris, France
}

Available online 16 February 2008

\begin{abstract}
Direct numerical simulations of two-dimensional decaying turbulence in wall bounded domains are presented. The Navier-Stokes equations are solved using a Fourier pseudo-spectral method with volume penalization. Starting from random initial conditions, we study the influence of the geometry of the domain on the flow dynamics, in particular on the long time behaviour. Circular, square, triangular and annular domains are considered and we show how the geometry plays a crucial role regarding the decay scenario towards final states. Three stages can be distinguished: formation of coherent vortices from random initial conditions, vortex wall interactions, and finally relaxation towards a quasisteady structure. The eigenvalues estimated from the decay rate of both energy and enstrophy depend on the geometry and agree well with the theoretical eigenvalues based on the Stokes mode of the corresponding domain. For the final states we find a linear functional relation between vorticity and streamfunction.
\end{abstract}

(C) 2008 Elsevier B.V. All rights reserved.

PACS: 47.27.Eq; 47.32.Cc

Keywords: 2D turbulence; Confined flows; Final states; Stokes eigenmodes

\section{Introduction}

Two-dimensional turbulence in wall-bounded domains has many applications in geophysical flows, e.g. the prediction of currents in oceanic basins, the transport and mixing of pollutants. Experiments in rotating tanks, e.g., in [2], leading to quasi two-dimensional geostrophic flows, have shown the formation of long-lived coherent vortices. Quasi two-dimensional experiments in stratified fluids for square and circular containers have been presented in $[10,6,16]$. Several numerical simulations of two-dimensional turbulence in bounded domains have been performed so far, e.g., in circular and square domains [15,5,4,22]. Compared to simulations in double periodic domains the decay scenario is altered in bounded domains with no-slip boundary conditions, since the

\footnotetext{
* Corresponding author. Tel.: +33 491118529; fax: +33 491113502.

E-mail addresses: kschneid@ cmi.univ-mrs.fr (K. Schneider), farge@1md.ens.fr (M. Farge).
}

role of viscous boundary layers is determinant, for a discussion see, e.g., [7].

The aim of the present paper is to study the influence of the geometry of the domain on the flow dynamics, in particular on its long-time behaviour. Therefore we consider four different geometries: circular, square, triangular and annular domains. Typically, we observe the formation of stable large-scale structures which persist for a long time before they are finally dissipated.

Late states of decaying two-dimensional flows in periodic domains were investigated, e.g., in $[17,23]$. Here we study the final states of wall-bounded flows considering different geometries with no-slip boundary conditions.

Several theoretical predictions of the long time behaviour of two-dimensional flows have been made for unbounded or periodic domains. Variational principles for predicting the final state are based on the 'selective decay' hypothesis supposing conservation of energy and decay of enstrophy [13]. In this heuristic approach enstrophy is minimized under constraint 
of conservation of energy and eventually additional conditions. Another variational hypothesis has been proposed, based on statistical mechanics, which introduces a measure of mixing, leading to the definition of an entropy. The final states then correspond to a maximum of entropy as turbulence maximizes mixing [8,19]. For two-dimensional flows in bounded domains, with either free-slip [9] or no-slip [10] boundary conditions, a different approach based on viscous eigenmodes of the Stokes flow has been used to predict the self-organization of the flow into 'final' states. Stokes eigenmodes in a square domain with no-slip boundary conditions have been computed in [14].

The paper is organized as follows. First, we briefly recall the volume penalization technique and the numerical method employed to solve Navier-Stokes equations in different geometries (Section 2). The construction of viscous eigenmodes is sketched in Section 3. Then, numerical results of decaying flows in four different geometries are presented in Section 4 and conclusions are drawn in Section 5.

\section{Numerical scheme and geometry}

The numerical technique we use here is based on a Fourier pseudo-spectral method with semi-implicit time discretization and adaptive time-stepping [21]. The Navier-Stokes equations are solved in a double periodic square domain of size $L=$ $2 \pi$ using the vorticity-velocity formulation. The bounded domain $\Omega$ is imbedded in a periodic domain and the noslip boundary conditions on the wall $\partial \Omega$ are imposed using a volume penalization method. A mathematical analysis of the method is given in [1], proving its convergence towards the Navier-Stokes equations with no-slip boundary conditions. Details on the code, together with its numerical validation, can be found in [21]. The governing equations in vorticity-velocity formulation, written in dimensionless form, are

$\partial_{t} \omega+\vec{u} \cdot \nabla \omega-v \nabla^{2} \omega+\nabla \times\left(\frac{1}{\eta} \chi \vec{u}\right)=0$,

where $\vec{u}$ is the divergence-free velocity field, i.e., $\nabla \cdot \vec{u}=0$, $\omega=\nabla \times \vec{u}$ the vorticity, $v$ the kinematic viscosity and $\chi(\vec{x})$ a mask function which is 0 inside the fluid, i.e., $\vec{x} \in \Omega$, and 1 inside the solid wall.

Four different geometries are considered: a circle with radius $R=2.8$, a square of sidelength $S=5.712$, an equilateral triangle with sidelength $T=5.8$ and an annulus with minor radius $R_{m}=0.8$ and major radius $R_{M}=2.8$. All domains are centred inside the periodic square domain of size $L=2 \pi$. The viscosity is set to $v=0.001$. For all computations the resolution is $N=256^{2}$ and the penalization parameter $\eta$ is chosen to be sufficiently small $\left(\eta=10^{-3}\right)$ [21].

Different integral quantities, the energy $E$, enstrophy $Z$ and palinstrophy $P$, can be defined as [11]

$E=\frac{1}{2} \int_{\Omega}|\vec{u}|^{2} \mathrm{~d} \vec{x}, \quad Z=\frac{1}{2} \int_{\Omega}|\omega|^{2} \mathrm{~d} \vec{x}$,

$P=\frac{1}{2} \int_{\Omega}|\nabla \omega|^{2} \mathrm{~d} \vec{x}$

respectively.
Table 1

Properties for different geometries

\begin{tabular}{lcccc}
\hline & Circle & Triangle & Annulus & Square \\
\hline$t_{\nu}$ & 200 & 180 & 210 & 280 \\
Theor. EV $\mu_{1}$ & 1.87 & - & 2.73 & 1.60 \\
Estim. EV & 1.89 & 5.88 & 2.74 & 1.70 \\
Estim. $\alpha$ & 1.90 & 4.25 & 2.70 & 1.70 \\
\hline
\end{tabular}

Viscous time, theoretical eigenvalues [10,12], eigenvalues estimated from the energy and enstrophy decay (Fig. 2) and estimated slope of the linear functional relationship at final instants (Fig. 4).

The energy dissipation is given by $\mathrm{d}_{t} E=-2 v Z$ and the enstrophy dissipation by

$\mathrm{d}_{t} Z=-2 v P+v \oint_{\partial \Omega} \omega(\vec{n} \cdot \nabla \omega) \mathrm{d} s$,

where $\vec{n}$ denotes the outer normal vector with respect to the boundary of the domain $\partial \Omega$. The line integral in (3) reflects the enstrophy production at the wall, involving the vorticity and its gradients, which is due to the no-slip boundary conditions. This second term is not present in the case of periodic boundary conditions.

\section{Viscous eigenmodes of the Stokes flow}

Final decay of two-dimensional turbulence in bounded domains with no-slip boundary conditions is characterized by a self-similar decay of the fundamental mode of the Stokes flow [5]. For a square domain an analytical expression was derived, either for stress-free [9], or for no-slip boundary conditions [10]. For the later case numerical computations of the Stokes eigenmodes and the corresponding eigenvalues were presented in [14]. The solution of the vorticity equation neglecting the nonlinear term

$\partial_{t} \omega-v \nabla^{2} \omega=0$

is expressed as a superposition of exponentially decaying modes, each characterized by an eigenvalue $\mu_{n}$,

$\omega(\vec{x}, t)=\sum_{\mu} C_{\mu} \omega_{\mu}(\vec{x}) \mathrm{e}^{-\mu_{n} v t}$,

with $\mu_{n}>0$, and where the constants $C_{\mu}$ are determined by the initial conditions. For each value of $\mu_{n}$ the following Helmholtz equation for $\omega_{\mu}(\vec{x})$ has to be solved.

$\nabla^{2} \omega_{\mu}(\vec{x})+\mu \omega_{\mu}(\vec{x})=0$.

Since for the vorticity no boundary condition is available we consider instead the streamfunction $\psi$. Replacing in Eq. (6) $\omega=\nabla^{2} \psi$, we obtain a fourth order PDE

$\nabla^{4} \psi_{\mu}(\vec{x})+\mu \nabla^{2} \psi_{\mu}(\vec{x})=0$.

The no-slip boundary condition of the velocity yields for the stream function $\psi=\frac{\partial \psi}{\partial n}=0$.

The available theoretical lowest eigenvalues for the circular, annular and square geometry are given in Table 1. For the circle the eigenvalue is the square of the first zero crossing of the 
Bessel function of order one, divided by the square of the radius $R$, to take into account the domain size. For the square domain, the value given in [10] has to be multiplied by $(\pi /(S / 2))^{2}$, which corresponds to the value computed numerically in [14] divided by $(S / 2)^{2}$, due to different normalizations. Analytical expressions for the Stokes eigenfunctions of the annular domain can be found in [12]. The corresponding eigenvalues are given by a transcendent equation containing Bessel functions of the first and second kind. More details on the eigenmodes of the circular and annular domains are given in the Appendix.

\section{Numerical results}

Starting with the same random initial conditions, i.e., a correlated Gaussian noise with an energy spectrum $E(k) \propto$ $k^{-4}$, we compute the flow evolution in the four different geometries for initial Reynolds numbers, $R e=2 D \sqrt{2 E} / \nu$, of about 1000 (where $D$ corresponds to the characteristic domain size). Fig. 1 shows the vorticity fields at early, intermediate and late times, for circular, square, triangular and annular domains. All flows organize into larger and larger scale structures until reaching the domain size and forming a structure which then no more evolves. For the circular geometry (Fig. 1, top) we observe the transition via a quasi-dipolar structure, before reaching the final state where a monopole is formed. It consists of a negative circular vortex surrounded by a band of positive vorticity which forms a kind of circular jet. The final state of the annular geometry (Fig. 1, bottom) corresponds to two ring-shaped bands of oppositely signed vorticity which corresponds to a circular jet. During the transition phase, a triangularly shaped vortical structure forms which is surrounded by three positive vortices. For the triangle and the square domain (Fig. 1, middle) we see that the final state is not yet completely reached. During the transition phase we observe a tripole which evolves towards a kind of circular jet as for the circular and annular domains. In the present simulations the infinite sequence of corner eddies of the Stokes eigenmodes, predicted by Moffatt [18] and computed numerically in [14], cannot be observed for the triangular and square domains. Indeed, the magnitude of these vortices decays exponentially and a high resolution spectral method where the basis functions satisfy the no-slip boundary conditions would be required for observing them.

Figs. 2 and 3 present the decay of different integral quantities, energy (Fig. 2, left), enstrophy (Fig. 2, right) and palinstrophy (Fig. 3, left) for the four geometries. All quantities exhibit at early times a rapid monotonuous decay, which is partly due to the fact that the flow has first to adjust to the boundary conditions, since the initial conditions do not satisfy them. For the square, circular and triangular geometries we observe an oscillatory behaviour in the palinstrophy decay, which is, however, less pronounced for the latter case. These oscillations are due to the enstrophy production at the wall. Considering the decay of the fundamental Stokes mode, we can characterize the long time decay of energy, enstrophy and palinstrophy, to be proportional to $\exp (-2 \mu \nu t)$ according to Eq. (5). At later times we find indeed, for all geometries and all quantities, an exponential decay behaviour for which
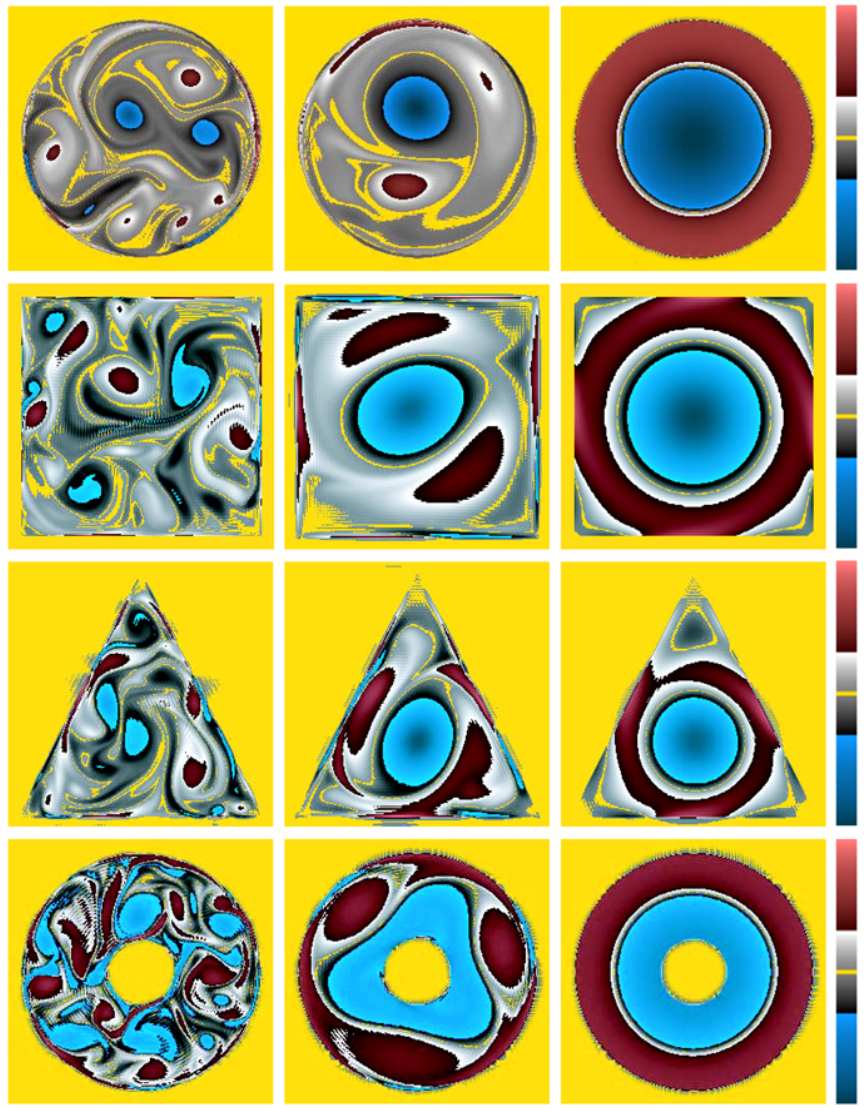

Fig. 1. 2d decaying turbulence in bounded domains. Vorticity fields at early (left), intermediate (middle) and late times (right). From top to bottom: circle, square, triangle and annulus.

the decay rates depend on the geometry. Table 1 presents the time instant $t_{v}$ when viscous decay starts to dominate for the different geometries. It is identified by considering the palinstrophy evolution and detecting the moment when the decay slows down and becomes exponential. We computed slopes by fitting an exponential curve using a least square method, applied to both energy and enstrophy evolution, which yield similar results. The square domain shows the slowest decay for all quantities, followed by the circle, the annulus, while the triangle exhibits the fastest decay. To get an estimation of the eigenvalue $\mu$ we divide the slopes thus obtained by twice the viscosity. The resulting estimated eigenvalues $\mu$ are given in Table 1 and are compared with the theoretical values based on the Stokes eigenmodes, given in [10] for the circle and the square geometry, and in [12] for the annulus. Note that the theoretical values are adapted to our normalization. The estimated eigenvalues agree well with the available theoretical values for all geometries.

The time evolution of the mean square wavenumber $k_{\lambda}=$ $\sqrt{Z / E}$, which is inversely proportional to the Taylor microscale $\lambda$, is plotted in Fig. 3, right. It is measuring the inverse average vortex size in the flow and is bounded from below by the size of the domain. For unbounded flows, one can show that $\frac{\mathrm{d} k_{\lambda}^{2}}{\mathrm{~d} t} \leq 0$, i.e., the average vortex size is monotonously increasing [17]. In the present cases we observe a monotonous decay at early times. At later times a nonmonotonous behaviour is found 

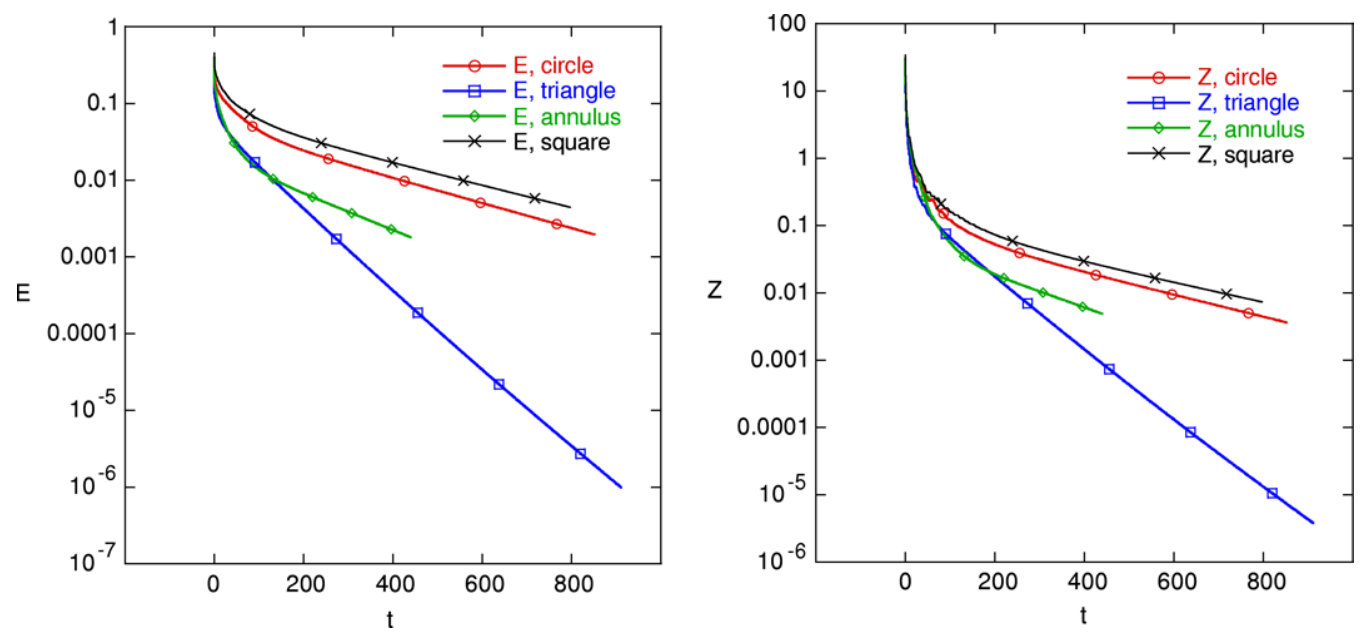

Fig. 2. Decay of energy $E(t)$ (left) and enstrophy $Z(t)$ (right) for circular, square, triangular and annular domains.
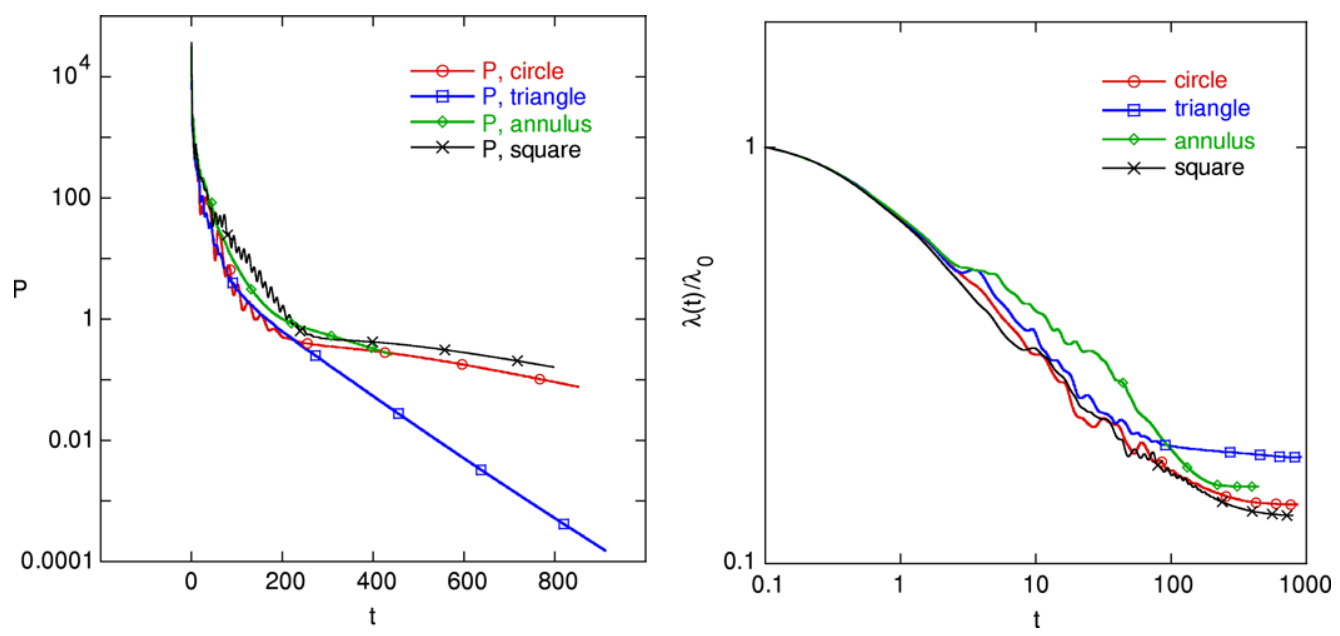

Fig. 3. Decay of palinstrophy $P(t)$ (left) and the normalized mean square wavenumber $k_{\lambda}(t) / k_{\lambda}(0)$ (right), for circular, square, triangular and annular domains.

which is due to the intermittent generation of vortices at the no-slip wall (cf. Fig. 3, right). Note that in [22] we also found a nonmonotonous behaviour for a circular domain at higher Reynolds number ( $R e=50$ 000). At late times, the mean square wavenumber becomes constant for all cases, which confirms that the size of the structure is not changing anymore. The coherence scatter plot, defined as the pointwise correlation between vorticity and stream function, is shown in Fig. 4 for the four geometries at the corresponding final instant of the simulations. The coherence plot measures the self-organization of the flow. A functional relationship between $\omega$ and $\psi$ implies that the nonlinearity has been depleted, and that the flow has reached a quasi-stationary state.

For the flows in bounded domains considered here we find a linear functional relationship between $\omega$ and $\psi$, i.e., $\omega=F(\psi)$ with $F(\psi)=\alpha \psi$ in the four cases. This is in agreement with the linear relationship found in [10] for the square domain. We also observe that close to the wall the linear relationship is less pronounced. For the triangular domain we still have some scattering which might be due to the persistence of higher order eigenmodes. The values of $\alpha$, obtained by fitting a straight line in the scatter plot (Fig. 4), are given in Table 1 and they agree approximately with the eigenvalues of the corresponding geometry.

\section{Conclusion}

By means of DNS of wall-bounded flows in domains of different geometries, we have shown that no-slip boundary conditions and the geometry of the domain play a crucial role for the decay of turbulent flows. At early times, we observe a decay of the flow which leads to self-organization and the emergence of vortices in the bulk flow, similarly to flows in periodic domains. At later times, larger scale structures form which depend on the domain geometry, and they finally relax towards quasi-steady states. The present results confirm both numerical and experimental studies performed for circular and square domains [16,7].

In contrast to simulations of two-dimensional turbulence in periodic domains, we do not observe selective decay in bounded domains with no slip boundary conditions, since in this case energy is no more conserved but strongly dissipated. The viscous dissipation becomes the dominant mechanism of these final states, which correspond to the fundamental Stokes eigenmodes of the different geometries. The nonlinear 

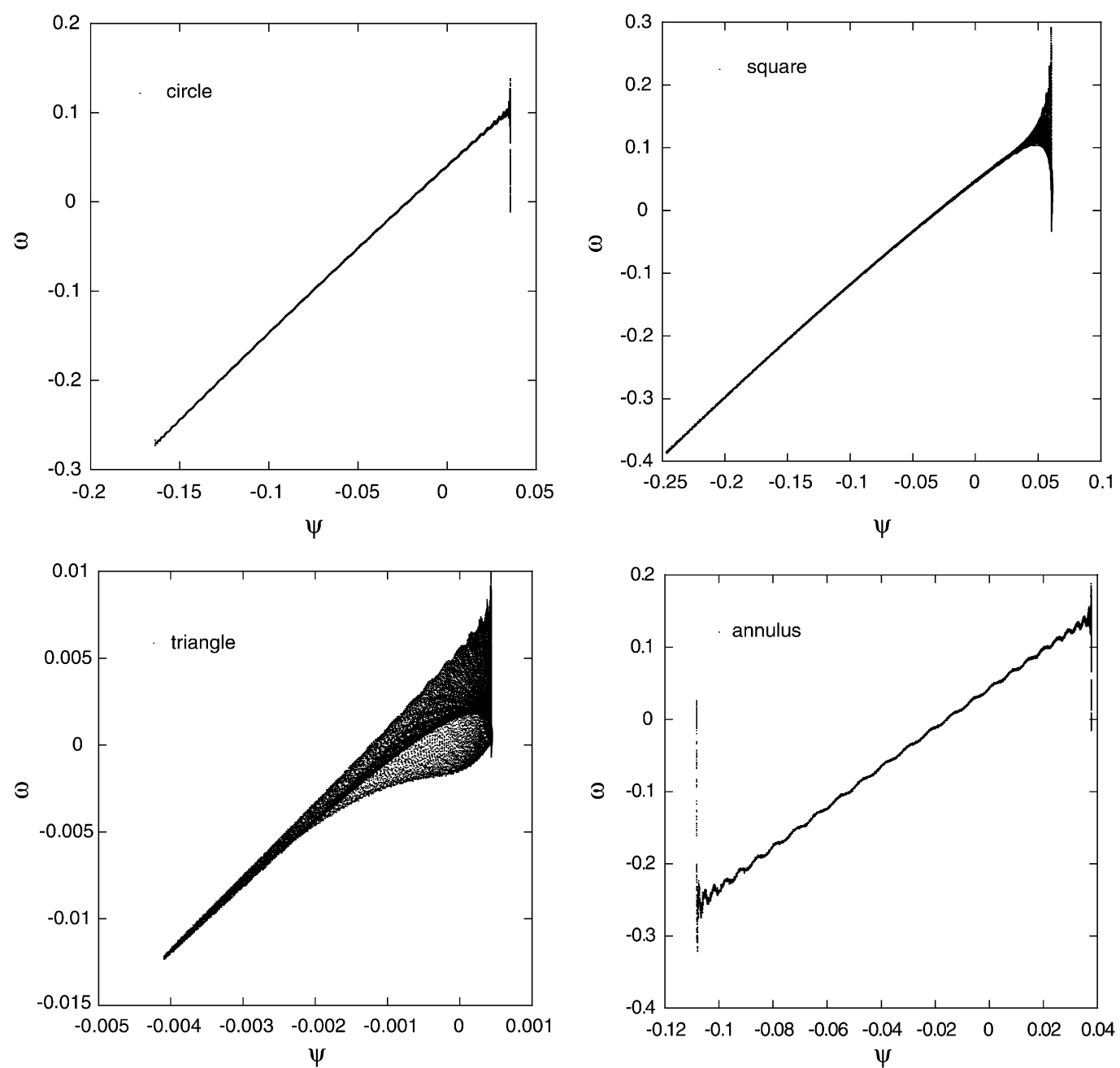

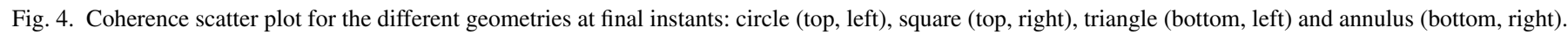

term in the Navier-Stokes equations is depleted and we observe a functional relationship between streamfunction and vorticity. For wall-bounded domains this relationship is linear, corresponding to the eigenmodes. This linear relationship, originally suggested by Batchelor [3], corresponds to steady motion of an inviscid fluid, or, when multiplied by $\exp (-\mu \nu t)$ to decaying motion of viscous fluid. The observed decay rates $\mu$ of the exponentially decaying energy and enstrophy agree well with the smallest eigenvalues of the Stokes eigenmodes of the different geometries.

\section{Acknowledgements}

We acknowledge financial support from the Agence Nationale de la Recherche, project "M2TFP". We thankfully acknowledge one of the referees for useful comments, and J. P. Kelliher for bringing to our knowledge Ref. [12].

\section{Appendix}

In the following we present the eigenfunctions and the corresponding eigenvalues for the circular and annular domains. Their derivation in velocity-pressure formulation can be found in the original papers $[20,12]$.
For the circular domain with radius $R$ the azimuthally symmetrical solutions of Eq. (7) are given by [20]

$\psi_{\mu}(r)=J_{0}\left(\sqrt{\mu_{n}} r\right)-J_{0}\left(\sqrt{\mu_{n}} R\right)$

with $r=|\vec{x}|$ and where $J_{0}$ denotes the Bessel function of first kind of order zero. The eigenvalues $\mu_{n}$ are obtained from the zeros of the Bessel function of first kind of order one, i.e.,

$J_{1}\left(\sqrt{\mu_{n}} R\right)=0$

which yields for the lowest eigenvalue, $\mu_{1}=1.873$ (with $R=2.8)$. Note that (8) satisfies $\psi_{\mu}(r=R)=0$ and $\partial_{r} \psi_{\mu}(r=R)=0$.

For the solution of the vorticity equation (5) we get correspondingly

$\omega(r, t)=\sum_{n} c_{n} \mu_{n} J_{0}\left(\sqrt{\mu_{n}} r\right) \mathrm{e}^{-\mu_{n} v t}$

For the annular domain with minor radius $R_{m}$ and major radius $R_{M}$ the azimuthally symmetrical solutions of Eq. (7) are given by [12]

$\psi_{\mu}(r)=J_{0}\left(\sqrt{\mu_{n}} r\right)-J_{0}\left(\sqrt{\mu_{n}} R_{M}\right)$ 


$$
-\frac{J_{1}\left(\sqrt{\mu_{n}} R_{m}\right)}{Y_{1}\left(\sqrt{\mu_{n}} R_{m}\right)}\left(Y_{0}\left(\sqrt{\mu_{n}} r\right)-Y_{0}\left(\sqrt{\mu_{n}} R_{M}\right)\right)
$$

where $Y_{0}$ and $Y_{1}$ denote the Bessel functions of second kind (also called Weber functions) of order 0 and 1 , respectively.

The eigenvalues $\mu_{n}$ are solutions of the transcendent equation

$$
J_{1}\left(\sqrt{\mu_{n}} R_{M}\right) Y_{1}\left(\sqrt{\mu_{n}} R_{m}\right)-J_{1}\left(\sqrt{\mu_{n}} R_{m}\right) Y_{1}\left(\sqrt{\mu_{n}} R_{M}\right)=0
$$

which yields (using Maple) for the lowest eigenvalue, $\mu_{1}=$ 2.731 (with $R_{m}=0.8$ and $R_{M}=2.8$ ).

For the vorticity in eq. (5) we get

$$
\begin{aligned}
\omega(r, t)= & \sum_{n} d_{n} \mu_{n}\left[J_{0}\left(\sqrt{\mu_{n}} r\right)\right. \\
& \left.-\frac{J_{1}\left(\sqrt{\mu_{n}} R_{m}\right)}{Y_{1}\left(\sqrt{\mu_{n}} R_{m}\right)} J_{0}\left(\sqrt{\mu_{n}} r\right)\right] \mathrm{e}^{-\mu_{n} v t} .
\end{aligned}
$$

For the square and triangular domains there are to our knowledge no explicit expressions available.

\section{References}

[1] P. Angot, C.H. Bruneau, P. Fabrie, Numer. Math. 81 (1999) 497-520.

[2] J. Aubert, S. Jung, H.L. Swinney, Geophys. Res. Lett. 29 (2002) 1876.
[3] G.K. Batchelor, An Introduction to Fluid Mechanics, Cambridge University Press, 1967. Reprinted 1994.

[4] H.J.H. Clercx, A.H. Nielsen, D.J. Torres, E.A. Coutsias, Eur. J. Mech. B Fluids 20 (2001) 557-576.

[5] H.J.H. Clercx, S.R. Maassen, G.J.F. van Heijst, Phys. Fluids 11 (3) (1999) 611-576.

[6] H.J.H. Clercx, S.R. Maassen, G.J.F. van Heijst, Phys. Rev. Lett. 80 (1998) 5129-5132.

[7] G.J.F. van Heijst, H.J.H. Clercx, D. Molenaar, J. Fluid Mech. 554 (2006) 411-431.

[8] G. Joyce, D. Montgomery, J. Plasma Phys. 10 (1973) 107.

[9] Y. Kondoh, M. Yoshizawa, A. Nakano, T. Yabe, Phys. Rev. E 54 (3) (1996) 3017-3020.

[10] J.A. van de Konijnenberg, J.B. Flor, G.J.F. van Heijst, Phys. Fluids 10 (3) (1998) 595-606.

[11] R.H. Kraichnan, D. Montgomery, Rep. Progr. Phys. 43 (1980) 547-619.

[12] D.-S. Lee, B. Rummler, Z. Angew. Math. Mech. 82 (6) (2002) 399-407.

[13] C.E. Leith, Phys. Fluids 27 (6) (1984) 1388-1395.

[14] E. Leriche, G. Labrosse, J. Comput. Phys. 200 (2004) 489-511.

[15] S. Li, D. Montgomery, B. Jones, Theoret. Comput. Fluid Dyn. 9 (1997) 167-181.

[16] S.R. Maassen, H.J.H. Clercx, G.J.F. van Heijst, Phys. Fluids 14 (2002) 2150-2169.

[17] W.H. Matthaeus, W.T. Stribling, D. Martinez, S. Oughton, Phys. Rev. Lett. 66 (1991) 2731-2734.

[18] H.K. Moffatt, J. Fluid Mech. 18 (1964) 1-18.

[19] R. Robert, J. Sommeria, J. Fluid Mech. 229 (1991) 291-310.

[20] B. Rummler, Z. Angew. Math. Mech. 77 (8) (1997) 619-627.

[21] K. Schneider, Comput. Fluids 34 (2005) 1223-1238.

[22] K. Schneider, M. Farge, Phys. Rev. Lett. 95 (2005) 244502.

[23] E. Segre, S. Kida, Fluid Dyn. Res. 23 (1998) 89-112. 